\title{
Light Bulb Moments in the Classroom: Probing Design Opportunities for Ambient LA Displays in Higher Education
}

\section{Pengcheng An ${ }^{1}$, Anouschka van Leeuwen ${ }^{2}$}

${ }^{1}$ Department of Education, Utrecht University, the Netherlands, ${ }^{2}$ Department of Computer Science, University of Waterloo, Canada.

\begin{abstract}
Teachers in higher education are tasked with the demanding job of providing support tailored to each individual student's need. To provide tailored support, teachers need to accurately monitor students' activities and decide on appropriate support interventions. Learning analytics applications have the potential to aid teachers to maintain an overview of their students' activities. However, those applications are often designed as centralized graphical displays, taking teachers' attention away from the classroom and sometimes overburdening teachers. Therefore, we investigate whether ambient Learning Analytics (LA) displays offer a solution to complement traditional LA applications, as these systems are designed as objects that integrate seamlessly into the classroom context. We conducted an exploratory study in Higher Education to investigate teachers' needs for information and their perception of ambient LA displays in relation to their teaching practice. We formulate three key findings and a set of design opportunities to inform future work of supporting the HE context with ambient LA displays.
\end{abstract}

Keywords: Ambient display; learning analytics; higher education; classroom; teacher; human-computer interaction. 


\section{Introduction}

Teachers in Higher Education (HE) are tasked with increasingly demanding jobs. They are expected to provide adaptive support to meet the needs of individual students. With a growing diversity in the student population, the combination of inside and outside of class activities, and less classroom hours than in primary and secondary education, providing adaptive support has become even more challenging. Several studies have described the complexity of scaffolding during tutorials, e.g., (Comas-Quinn, 2011; Greenberg et al., 2011; Roehl et al., 2013; Vaughan, 2007). Teachers often experience time pressure and "a feeling that learning was too distributed, that there were too many places to check and contribute to" (Comas-Quinn, 2011) p. 227).

Emerging innovations in learning analytics (LA) have been proposed to aid teachers by offering aggregated insights into students' activities and potential needs for support. Although these studies generally show that the offered information is deemed relevant by teachers, the actionability of the information is sometimes low (Van Leeuwen, 2019; Wise \& Jung, 2019). This is because classroom sessions are filled with intensive interpersonal interactions with learners, and it is taxing for teachers to access the LA applications on top of their already dynamic practice. A human-centered design approach is therefore needed to make LA information more seamlessly integrated into teachers' classroom workflow. In the domain of human-computer interaction, a number of explorations have been done under the notion of ambient information system, or peripheral display (An et al., 2020). The idea is to create glanceable, relatively less-detailed displays as part of the classroom environment to enhance teachers' awareness of the situation without demanding their focal engagement.

For instance, ClassBeacons (An et al., 2019) portrays classroom proxemics data (Figure 1 Left): each ambient lamp gradually turns from yellow to green as the teachers cumulatively spends time around it, facilitating teachers to dynamically plan how to distribute time and attention over students. FireFlies-VLE (d'Anjou et al., 2019) uses ambient lamps to convey students' progress in exercises on their computers, based on real-time LA data, to ease teachers' monitoring of learning processes and their awareness about students' potential needs (Figure 1 Right). These ambient information systems have been found to meaningfully support teachers and learners in classroom activities: e.g., in supporting teachers to keep track of what is going on around and make data-informed decisions without imposing excessive mental burden. However, most of such examples have been targeted at primary or secondary classrooms. Much less is known about the potential and design implications of ambient LA displays for supporting teachers in higher education. 


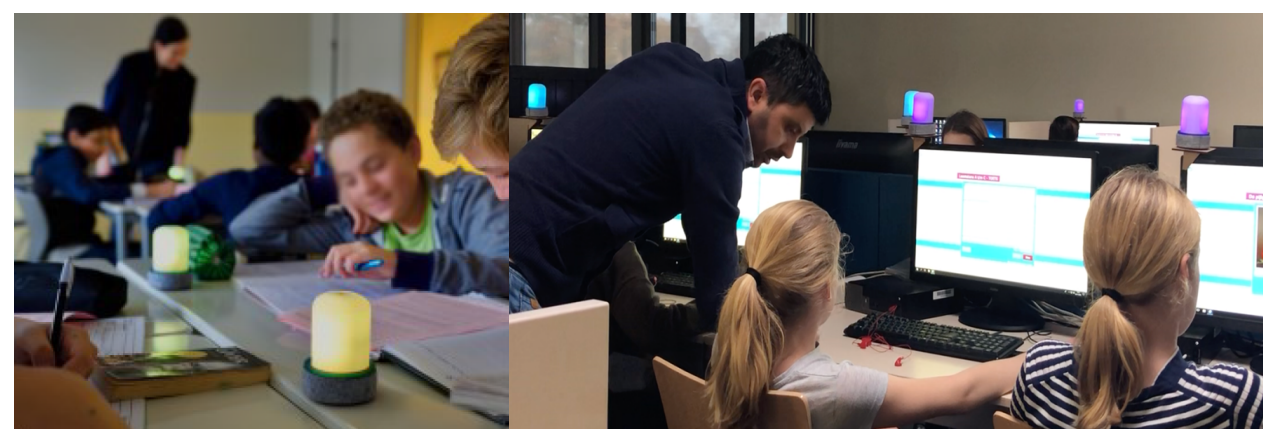

Figure 1. Examples of ambient LA displays in the SE context: ClassBeacons (Left), FireFlies-VLE (Right).

The current paper therefore aims to shed light on the potential role of ambient LA displays in HE. We compare earlier work in secondary education (SE) to an exploratory study in HE to better understand this relatively less addressed design context. We present several design opportunities that can serve as a research agenda for future studies in this field.

\section{Method}

Based on earlier work in secondary education, we investigated the HE context to identify teachers' needs for information during tutorials and discuss opportunities and implications for supporting HE teachers with ambient LA displays. We compared the results of the empirical studies in secondary education (An et al., 2019; Anjou et al., 2019) to this study in $\mathrm{HE}$, allowing us to formulate specific design recommendations for the HE context. This paper can thus best be characterized as a cross-case analysis (Miles \& Huberman, 1994).

\subsection{Context and participants}

The context of the study was an undergraduate course on the topic educational design. The course consisted of weekly plenary lectures and tutorials. The course was assessed by an individual exam and a collaborative assignment (performed in groups of 4). Students were expected to prepare for the tutorials by creating multiple choice questions about the course materials, which they submitted through PeerWise. Subsequently, all students in the course can practice with the student-generated questions, and the activity thus serves the dual goal of processing the materials as well as to jointly create a database of questions to practice with. During the tutorial, time is spent on discussing the student-generated questions and any other questions students may have about the materials. Also, the tutorials are meant for receiving teacher supervision on the collaborative assignment. The total number of students in the course was 150 . They were divided into groups of about 25 students for the tutorials. The teaching team consisted of 7 teachers, each supervising one or two of the tutorial groups. The project ran for several cohorts, starting with the introduction of a non-ambient learning analytics system, and continuing with investigating the potential role of ambient systems. 
The results of the first step are reported in (Van Leeuwen, 2019). In the results section, we will sometimes refer to some of these findings because they were the basis for the second step of investigating the potential for ambient displays.

\subsection{Procedure and instruments}

We conducted a user design and evaluation study to examine teachers' need for information and their reaction to a prototype ambient display. Because of the Covid-19 pandemic, the final step of testing the ambient display during an actual tutorial could unfortunately not be performed. The data collection consisted of two parts. First, we performed a contextual inquiry, meaning that we observed a tutorial and interviewed one of the teachers afterwards (female, more than 5 years teaching experience). The observation and interview were meant to probe how the tutorial takes place specifically in terms of the potential role for an ambient display. Thus, it focused on how the teacher and students walked around, what kind of questions teachers and students asked, what kind of cues were available to the teacher to estimate students, etc. The observation and interview took about 4 hours. Second, a co-design brainstorm session was held with 3 of the teachers in the course ( 2 female, 2 teachers with less than 5 years of teaching experience). In this session, the teachers were familiarized with the idea of FireFlies, a set of ambient lamps for displaying glanceable LA information, see Figure 2. They were asked about their preferences for what type of information to visualize, and for their suggestions about how to visualize that data. They were also asked to explain their choices in light of their teaching practice in this specific course. This session took about 1.5 hour. From these two types of sessions, a summary was made about design considerations for ambient displays for the HE context. This summary was contrasted with the secondary education context via a cross-case analysis. The most noteworthy similarities and differences were distilled and are discussed in the section below.

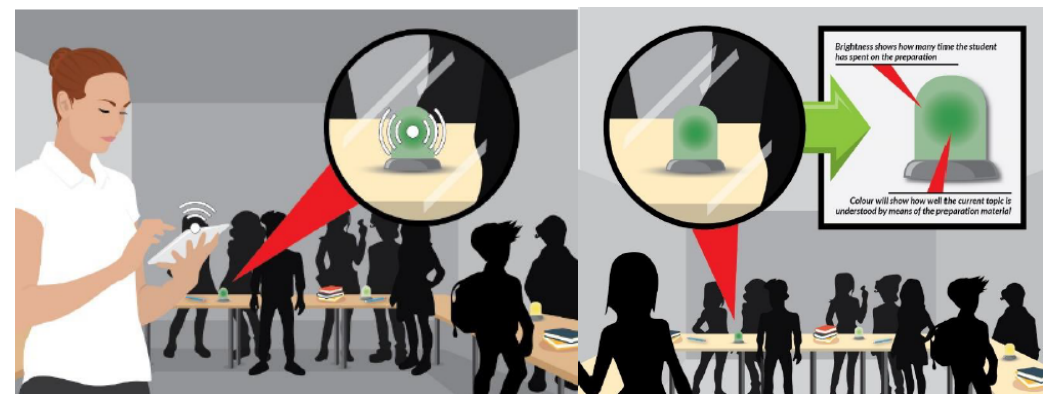

Figure 2. Images used for explaining the ambient LA concept to the teachers (by Teuwen, Thijssen \& van de Ven). 


\section{Findings: HE teachers' needs and resulting design opportunities}

\subsection{Timeliness of the provided information and flexibility in the designed tool}

A similarity between secondary and higher education is that teachers need information in a timely manner, so that they could still use the information on the fly to modify their practices, rather than only reflect on prior sessions. LA reports used in the first iteration of the project (Van Leeuwen, 2019) "came too late", as they were sent "a week later" from the tutorial session that the data were derived from. Another HE teacher mentioned that "just-in-time information" is needed, so that the information could more directly benefit their tutorial instead of only triggering retrospective analysis. Similarly, SE teachers in prior cases appreciated the deployed systems for offering on-the-spot LA information which is relevant to their ongoing activity (Anjou et al., 2019).

Another important similarity between the two groups of teachers is their need for flexibility and customization in the designed ambient information systems. Teachers differed in their reported practices. Some teachers for example divided their time equally among all students (e.g., using an alarm clock to keep track of the time), while others more intuitively allocated their attention to where it was needed most (see similar results in the SE case (An et al., 2019)). In line with that finding, there were also differing views on how useful a certain design solution would be, and how teachers would use it in their own classroom. As suggested in both contexts, such differing of opinions could be related to personal factors like teachers' levels of teaching experience, technology acceptance, or their different teaching styles. For example, one HE teacher said that when she was a beginning teacher, she would have liked to know what to expect in each week and which parts of the course would be challenging for students. But that information became less relevant as she had more experience.

Design opportunities: HE and SE teachers' similar need for timely information during teaching confirms the potential of developing ambient LA displays for the HE context. The personal nature of teachers' needs suggests that developers could afford flexible use or customizable features in the ambient information tool to better adapt to teachers with different experiences or teaching styles (e.g., by allowing them to configure certain display rules or features according to their preferences or professional beliefs).

\subsection{Characteristics of the student population}

Substantial differences of teachers' needs for ambient LA displays between the SE and HE contexts could exist due to the differences we found in teachers' descriptions of the student population. First, the learning goals and processes of HE students features much more flexibility and autonomy. One HE teacher emphasized that "students are responsible" for determining their learning process, and "with the structure of this course we offer that 
freedom". As students have more control over when and at what pace to study course materials (Condie \& Livingston, 2007), for teachers, extra cognitive load is demanded in monitoring the amount of time and the type of activities students engage in (Abeysekera $\&$ Dawson, 2015; Yen \& Lee, 2011), and the differences between students might be larger than in other contexts. This thus requires a visualization that is able to distinguish information for different learners on a fine-grained level. We also found that students' help-seeking behaviors and attitudes in HE are different, which result in different design rationales for creating appropriate social awareness in the classroom. Prior studies showed that ambient displays contributed to student accountability and peer awareness, but careful design was needed to avoid undesirable social effects by putting students on the spot (Anjou et al., 2019). We found that students in HE, especially more experienced part-time students that may have jobs, are less subject to these social effects and are more at ease with indicating their needs for support, whereas the younger students are not.

Design opportunities: This finding implies that an ambient LA display could benefit HE teachers considerably if its information facilitates teachers to monitor and differentiate the diverse learning processes of students (e.g., by indicating what they are working on and how far they have gone). This finding also implies that when designing the social awareness of an ambient LA displays for an HE classroom, developers may need to address differing attitudes or preferences from multiple student groups (e.g., by providing teachers, as well as students, with controls over how and when to make the information visible).

\subsection{Type of information and type of inferences}

Another difference was found between SE and HE teachers in terms of what type of information is most relevant to have during classroom sessions. The SE teachers in prior studies appreciated the ambient displays based on in-class data (An et al., 2019; d'Anjou et al., 2019) or student inputs (Sellier \& An, 2020)), whereas the HE teachers emphasized the value of combining outside-class data to support their in-class practice ("knowing how students prepared helps me prepare for a session”). As reported, these outside-class data could concern students' level of preparation, challenging parts found in the video lecture, group collaboration progress, etc. This difference is caused partly by the adoption of blended learning and the dominant format of HE lessons which has been changed from traditional lecture to face-to-face guided practice (Staker \& Horn, 2012). As a result, to better support students who learn at their own pace and have differing prior knowledge, HE teachers need rich insights into learners' outside-class processes so that they could act adaptively in the classroom. It is thus implied that ambient LA design for HE could put extra focus on supporting teachers' in-class decisions by offering them insights based on outside-class LA data (e.g., visualize students' status, progress, or inputs gathered from their outside-class sessions). A related observed difference is that HE teachers might need information support that has a higher level of inferences of the LA data. As aforementioned, the flexibility and 
autonomy of HE learning processes makes it difficult for teachers to identify learners' actual needs. Teachers need to interpret data from outside-class activities and use it to inform inclass decisions. The type of inferences that teachers need to make are thus quite complex. Some examples teachers gave of this complexity concern the possible problem occurring in team collaboration, or potential difficulties or misconceptions of students.

Design opportunities: These examples suggest that, in order to generate actionable insights, HE teachers need information that is aggregated from multiple LA indicators (e.g., from both their outside-class activities and in-class performances), with relatively higher level translations from the raw data. While the prior cases in SE showed that sometimes a lowlevel data portrayal would be useful enough for the SE teachers to skillfully interpret the classroom situations, our current study suggests that HE teachers may need ambient LA displays that are based on higher-level LA constructs or indicators based on multiple constructs, or that they need recommendations for actions.

\section{Conclusion}

Teachers in HE are tasked with the demanding job of providing support tailored to each individual student's need. Most learning analytics applications for teachers rely on centralized, focal displays. In this paper, we explored the potential of ambient LA displays that are designed to seamlessly integrate into the classroom context. We conducted an exploratory study in HE to investigate teachers' needs for information and their perception of ambient displays in relation to their teaching practice. Our results show that teachers' need for information differs fundamentally in comparison to the secondary education context. Most notably, the characteristics of HE students, their increased level of autonomy, and the fact that a large part of learning processes take place outside classrooms, mean that teachers need timely insights in individuals' trajectories during classroom sessions. From these results, we formulated three design opportunities to serve as a research agenda for exploring the potential of ambient LA displays in Higher Education.

\section{Acknowledgements}

We thank P.H.A. Teuwen, Y.J. Thijssen, and N.T. van de Ven for helping in the research and preparing the visual materials. We thank all the involved teachers and students. Our thanks also go to Dr. Bakker, Prof. Eggen, and Dr. Chuang who have supported this work.

\section{References}

Abeysekera, L., \& Dawson, P. (2015). Motivation and cognitive load in the flipped classroom: definition, rationale and a call for research. Higher Education Research \& 
Development, 34(1), 1-14.

An, P., Bakker, S., Ordanovski, S., Taconis, R., Paffen, C. L. E., \& Eggen, B. (2019). Unobtrusively Enhancing Reflection-in-Action of Teachers through Spatially Distributed Ambient Information. Proceedings of the 2019 CHI Conference on Human Factors in Computing Systems - CHI '19, 1-14. https://doi.org/10.1145/3290605.3300321

An, P., Holstein, K., D’Anjou, B., Eggen, B., \& Bakker, S. (2020). The TA Framework: Designing Real-time Teaching Augmentation for K-12 Classrooms. CHI '20: Proceedings of the 2020 CHI Conference on Human Factors in Computing Systems, 117. https://doi.org/10.1145/3313831.3376277

d'Anjou, B., Bakker, S., An, P., \& Bekker, T. (2019). How peripheral data visualisation systems support secondary school teachers during VLE-supported lessons. DIS 2019 Proceedings of the 2019 ACM Designing Interactive Systems Conference, 859-870. https://doi.org/10.1145/3322276.3322365

Bloom, B. S. (1956). Taxonomy of Educational Objectives. New York: David McKay.

Comas-Quinn, A. (2011). Learning to teach online or learning to become an online teacher: An exploration of teachers' experiences in a blended learning course. ReCALL, 23(03), 218-232.

Condie, R., \& Livingston, K. (2007). Blending online learning with traditional approaches: changing practices. British Journal of Educational Technology, 38(2), 337-348.

Domenech, J., Blazquez, D., de la Poza, E., \& Muñoz-Miquel, A. (2015). Exploring the impact of cumulative testing on academic performance of undergraduate students in Spain. Educational Assessment, Evaluation and Accountability, 27(2), 153-169. doi: 10.1007/s11092-014-9208-z.

Greenberg, J., Pomerance, L., \& Walsh, K. (2011). Student Teaching in the United States. National Council on Teacher Quality.

Miles, M. B., \& Huberman, A. M. (1994). Qualitative data analysis: An expanded sourcebook. sage.

Roehl, A., Reddy, S. L., \& Shannon, G. J. (2013). The flipped classroom: An opportunity to engage millennial students through active learning strategies. Journal of Family \& Consumer Sciences, 105(2), 44-49.

Sellier, N., \& An, P. (2020). How Peripheral Interactive Systems Can Support Teachers with Differentiated Instruction. Proceedings of the 2020 ACM Designing Interactive Systems Conference, 1117-1129. https://doi.org/10.1145/3357236.3395497

Staker, H., \& Horn, M. B. (2012). Classifying K-12 blended learning. Innosight Institute.

Van Goch, M. (2018). Creativity in liberal education before and after study commencement. 4th International Conference on Higher Education Advances (HEAd'18), 1475-1483. doi: 10.4995/HEAd18.2018.8228.

Van Leeuwen, A. (2019). Teachers' perceptions of the usability of learning analytics reports in a flipped university course: when and how does information become actionable knowledge? Educational Technology Research and Development, 67(5), 1043-1064. https://doi.org/10.1007/s11423-018-09639-y 
Van Vugt, M., Hogan, R., \& Kaiser, R. B. (2008). Leadership, followership, and evolution: Some lessons from the past. American Psychologist, 63(3), 182-196. doi: 10.1037/0003066X.63.3.182.

Vaughan, N. (2007). Perspectives on blended learning in higher education. International Journal on E-Learning, 6(1), 81-94.

Wise, A. F., \& Jung, Y. (2019). Teaching with analytics: Towards a situated model of instructional decision-making. Journal of Learning Analytics, 6(2), 53-69.

Yen, J.-C., \& Lee, C.-Y. (2011). Exploring problem solving patterns and their impact on learning achievement in a blended learning environment. Computers \& Education, 56(1), 138-145. 